\title{
DESARROLLO DE UN SISTEMA DE CRÍA ARTIFICIAL PARA ANASTREPHA FRATERCULUS (WIED.) (DIPTERA: TEPHRITIDAE)
}

\author{
HECTOR S. FLORES, ${ }^{1}$ EMILIO HERNÁNDEZ ${ }^{1}$ \& JORGE TOLEDO ${ }^{2}$ \\ ${ }^{1}$ Programa Moscafrut SAGARPA-IICA. Camino a Cacaotales S/N. Metapa de Dominguez, Chiapas, \\ C.P. 30,860. México. Tel. \& Fax: (52-962) 6435059. <emilio.hernandez@iica-moscafrut.org.mx> \\ ${ }^{2}$ Departamento de Entomología Tropical. El Colegio de la Frontera Sur. Apartado Postal 36. \\ Tapachula, Chiapas, 30,700 México.
}

Flores, H. S., E. Hernández \& J. Toledo. 2012. Desarrollo de un Sistema de Cría Artificial para Anastrepha fraterculus (Wied.) (Diptera: Tephritidae). Acta Zoológica Mexicana (n. s.), 28(2): 321-340.

RESUMEN. Se describe el proceso metodológico para solucionar dos cuellos de botella que dificultan el establecimiento de la cría artificial de Anastrepha fraterculus a gran escala. Primero, la selección del sistema de oviposición y segundo la adaptación a la dieta artificial utilizada para el desarrollo larvario. Las hembras tuvieron una mayor preferencia para ovipositar en esferas de color amarillo y en paneles planos elaborados con tela tipo "organza” de color blanco cubiertos con una película de silicón transparente. Después de evaluar las dietas para el desarrollo larvario de cinco especies de moscas de la fruta, la dieta con la que se obtuvieron los rendimientos más altos correspondió a la dieta ALU (Anastrepha ludens), con la cual la supervivencia de huevo a larva fue del 26.9\%, peso de larva de $19.6 \mathrm{mg}$, porcentaje de pupación a las 24 h $97.7 \%$, peso de pupa 16.7 mg y emergencia de adultos de $83.3 \%$. El análisis de las curvas de supervivencia $\left(\mathrm{n}_{\mathrm{x}}, \mathrm{l}_{\mathrm{x}}\right)$ y la estimación de la expectativa de vida $\left(\mathrm{e}_{\mathrm{x}}\right)$ indicaron que al menos el $50 \%$ de los individuos vivieron $\sim 40$ días. La expectativa de vida del adulto al momento de la emergencia fue de 38.74 días. La tasa neta de reproducción fue de 345.38 hijas/madre/generación, la tasa intrínseca de crecimiento poblacional fue de 0.35 días $^{-1}$; mientras que el tiempo medio de generación y el tiempo de duplicación de la población fue de 16.82 y de 20.2 días, respectivamente. Asimismo, se discuten valores de los parámetros de calidad registrados en los insectos luego de 16 generaciones bajo condiciones de cría artificial.

Palabras clave: Cría de insectos, dieta larvaria, mosca sudamericana de la fruta, sustrato artificial para colecta de huevo.

Flores, H. S., E. Hernández \& J. Toledo. 2012. Development of an artificial mass-rearing method for Anastrepha fraterculus (Wied.) (Diptera: Tephritidae). Acta Zoológica Mexicana (n. s.), 28(2): 321-340.

ABSTRACT. A methodological process is described here to solve two bottle necks that render the establishment of fruit flies under artificial conditions for mass production difficult. The first was the

Recibido: 09/06/2011; aceptado: 25/04/2012. 
selection of an oviposition system and the second the adaptation to the artificial diet used for larval development. Females preferred to oviposit on yellow spheres, and on white organza cloth flat panels covered with transparent silicon. A larval diet used to rear A.ludens, allowed the highest larval development, survival from egg to larva was $26.9 \%$, larval weight $19.6 \mathrm{mg}$, pupation at $24 \mathrm{~h} 97.7 \%$, pupal weight $16.7 \mathrm{mg}$ and adult emergence $83.3 \%$. Survival curves $\left(\mathrm{n}_{\mathrm{x}}, 1_{\mathrm{x}}\right)$ and life expectancy $\left(\mathrm{e}_{\mathrm{x}}\right)$ indicated at least $50 \%$ of the adults survive $\sim 40$ days. Life expectancy was of 34.74 days. The reproductive rate was 345.38 daughters/mother/generation; the intrinsic rate of population growth was 0.35 days $^{-1}$; while the median generation time and the time of duplication were 16.82 and 20.2 days, respectively. Moreover, we discuss results based on quality control parameters of insects after of 16 generations in mass-rearing under artificial conditions.

Key words: Mass-rearing, larval diet, South American fruit fly, artificial egg collection.

\section{INTRODUCCIÓN}

En México la mosca Sudamericana de la fruta, Anastrepha fraterculus (Wied.) (Diptera: Tephritidae) es un plaga de importancia secundaria debido a que se localiza en la región sureste del país donde la guayaba (Psidium guajava L.) se cultiva a baja escala o como árbol de traspatio (Celedonio-Hurtado et al. 1995); sin embargo, en Argentina, Brasil y Perú representa una severa plaga del mango, manzana, cítricos y guayaba entre otros cultivos (Ortiz 1999). Por este motivo, existe un gran interés por un mayor conocimiento de su biología para desarrollar programas de manejo integrado utilizando la Técnica del Insecto Estéril (TIE) como elemento clave (González et al. 1971, Jaldo et al. 2001, Ortiz 1999). El primer paso para aplicar la TIE, es establecer una colonia para cría, solucionando factores que dificulten su implementación como son: 1) selección del sistema de oviposición, y 2) adaptación de los insectos a una dieta para el desarrollo larvario (Rössler 1975, Boller \& Chambers 1977, Leppla et al. 1983).

El sistema de oviposición y colecta de huevos debe ser efectivo, durable, de fácil construcción y de mantenimiento sencillo (Prokopy \& Boller 1970). La metodología general para su desarrollo consiste en iniciar evaluando los sustratos utilizados para moscas del mismo género o de la misma familia que tengan comportamiento similar de oviposición (Hernández et al. 2004), además de considerar otros aspectos como el color y la forma. Para la colonización y cría masiva de moscas de la fruta se ha utilizado una gran diversidad de dispositivos, como los denominados "medias naranjas" elaborados con tela tipo "gasa” cubierta con parafina teñida de color rojo para el caso de la mosca Mexicana de la fruta, Anastrepha ludens (Loew) (McPhail \& Guiza 1956) y teñidos de negro para Rhagoletis pomonella (Walsh) (Prokopy 1966), receptáculos perforados de color verde para Ceratitis capitata Wied. (Tanaka 1965), esferas de agar envueltas con membrana de papel parafilm para $R$. cerasi (L.) (Boller 1968), tela tipo "lino" de color negro para la cría masiva de A. ludens, tela tipo "organza" de color blanco para A. obliqua, y tela tipo "satin" color azul para A. serpentina (Domínguez et al. 2010). 
En lo que respecta a la dieta de larvas, los distintos ingredientes así como las proporciones con que se formulan son factores decisivos al momento de su elaboración. El agua es el ingrediente de mayor proporción y funciona como vehículo para que las larvas obtengan los nutrientes requeridos durante su desarrollo. Seguida de los texturizantes, cuya proporción va del 1 al 26\% en la formulación de las dietas, donde los ingredientes más utilizados son: polvo de olote, bagazo de betabel, bagazo de caña y soya texturizada (Schwarz et al. 1985, Stevens 1991, Artiaga-López et al. 2004). Algunos texturizantes como la harina de soya texturizada, harina de maíz y salvado de trigo, aportan también grasas y lípidos. La sacarosa es la principal fuente de carbohidratos y contribuye para la formación de la estructura y función de los tejidos (Message \& Zucoloto 1989, Fontellas \& Zucoloto 2003). La principal fuente de proteína son las levaduras secas inactivas, las cuales contienen amonio, urea y algunos aminoácidos para sintetizar las vitaminas del grupo B requeridos para la reconstrucción y regeneración de órganos y tejidos de los insectos (Vargas et al. 1994, Domínguez et al. 2010). Las vitaminas del complejo B como la tiamina, riboflavina, ácido nicotínico y pantoténico son esenciales para un buen desarrollo de los adultos como se reporta en Bactrocera curcubitae (Coquillet); además, en las hembras contribuye en la producción de huevos y en machos aumenta la viabilidad del esperma (Dadd 1973). Además de estos factores, es importante considerar la consistencia y palatabilidad obtenidas de la mezcla de los ingredientes, lo cual impacta en la textura final de la dieta y finalmente en el desarrollo de la larva (Singh 1984).

Debido a la ausencia de referencias para la cría artificial de $A$. fraterculus para poblaciones nativas de México y tomando como referencia los métodos para colonización y cría masiva de moscas de la fruta en la Planta Moscamed-Moscafrut (SAGARPA-IICA) desarrollados para la oviposición y dietas para el desarrollo larvario de las moscas de la fruta, A. ludens y A. obliqua (Domínguez et al. 2010); el objetivo de esta investigación fue identificar un sustrato de oviposición adecuado y evaluar distintas dietas para larvas con la finalidad de brindar los lineamientos básicos para el desarrollo de protocolos de cría masiva de la mosca Sudamericana de la fruta, $A$. fraterculus. Asimismo se estimaron parámetros demográficos y se verificó la estabilidad para los parámetros de calidad y demográficos del insecto a través de 16 generaciones.

\section{MATERIALES Y MÉTODOS}

Material Biológico. El material biológico para la colonización se obtuvo en estado de larva a partir de frutos de guayaba (Psidium guajava L.) infestados que fueron colectados en el municipio de Unión Juárez, Chiapas. Los frutos colectados fueron trasladados al laboratorio de moscas de la fruta de El Colegio de la Frontera Sur, Unidad Tapachula, en donde fueron contados y pesados en una balanza granataria 
(Ohaus, Pine Brook, NJ); después fueron colocados en charolas de plástico (55 cm $\times 45 \mathrm{~cm} \times 12 \mathrm{~cm}$ ) en donde permanecieron seis días bajo condiciones ambientales de $26 \pm 1^{\circ} \mathrm{C}, 75 \pm 5 \%$ de H.R. y un fotoperiodo de 12:12 luz:oscuridad, hasta que las larvas alcanzaron el tercer estadio. Posteriormente los frutos fueron abiertos para extraer las larvas, las cuales fueron colocadas en contenedores de plástico $(10 \mathrm{~cm}$ de ancho $\times 12 \mathrm{~cm}$ de alto $\times 20 \mathrm{~cm}$ de longitud) con vermiculita húmeda para promover la pupación. Las pupas permanecieron en los contenedores por 13 días (a las condiciones ambientales indicadas previamente) y después fueron separadas de la vermiculita con un tamiz (Malla 14) y trasladadas al Laboratorio de Colonización y Cría de la Subdirección de Desarrollo de Métodos, Programa Moscafrut SAGARPA-IICA, ubicado en Metapa de Domínguez, Chiapas, México, donde se desarrolló este trabajo. El material se mantuvo durante cinco generaciones infestando frutos de guayaba hasta lograr una colonia pura de $A$. fraterculus, eliminando individuos de Anastrepha obliqua y A. striata. Los adultos se mantuvieron en condiciones de laboratorio de $26 \pm 1$ ${ }^{\circ} \mathrm{C}$ y $60 \pm 10 \%$ de humedad relativa y fotoperiodo de 10 horas oscuridad por $14 \mathrm{~h}$ de iluminación. La iluminación correspondió a un promedio de 800 lux en el interior de jaulas de plexiglass de $30 \times 30 \times 40 \mathrm{~cm}$. Al mismo tiempo el número de individuos incremento de una generación a otra hasta contar con suficiente cantidad para iniciar pruebas con sustratos para oviposición y dietas para el desarrollo de la larva.

Selección del Color del Sustrato de Oviposición. En este estudio se evaluó el efecto del color del sustrato de oviposición sobre el volumen de huevo puesto por las hembras. Como sustrato de oviposición se utilizaron esferas hechas usando $30 \mathrm{~g}$ de fucelleron (Burtonite 44, Tic Gums; Inc. Madison, U.S.A) en $1 \mathrm{~L}$ de agua que se calentó por 30 minutos a punto de ebullición $\left(80^{\circ} \mathrm{C}\right)$. Para teñir las esferas, se agregó $1.5 \mathrm{ml}$ de colorante vegetal (McCormick). Los colores de las esferas evaluadas fueron verde (Pantone 16-6339), rojo (Pantone 17-1563), negro, azul (Pantone 18-4051), amarillo (Pantone 12-0738) (Eiseman \& Herbert 1990) y esferas que no tenían color (transparentes). Posteriormente, la mezcla se vació en moldes para esferas con 2.54 cm de diámetro y una vez que estuvieron frías se envolvieron con una membrana de papel parafilm (American Nacional Can TM, Nena, Wi). Cada color fue considerado un tratamiento, de esta forma se tuvieron seis tratamientos, cada uno con 10 repeticiones, en un diseño completamente al azar, lo que dio un total de 60 unidades experimentales. Cada unidad experimental consistió en una jaula de vidrio de $30 \times 30$ $\times 30 \mathrm{~cm}$ con cinco parejas, las cuales fueron acondicionadas al momento de la emergencia de los adultos. Los adultos evaluados en este estudio correspondieron a la 6a. generación, los cuales fueron alimentados con una proporción 3:1 de azúcar: proteína hidrolizada (ICN Biomedicals, Costa Mesa, CA) (Message \& Zucoloto 1989) y el agua se les proporcionó en viales de vidrio cubiertos con torundas de algodón.

Las esferas fueron colgadas de la parte superior de cada jaula de acuerdo al color del tratamiento que le correspondió y fueron remplazadas cada 24 h. Esta actividad 
se realizó durante 10 días consecutivos, considerando cada día como una co-variable. Para extraer los huevecillos, las esferas fueron cortadas en pequeñas rebanadas y colocadas en agua para ser disueltas mediante un sistema de burbujeo producido por el flujo de aire de una bomba de acuario (3 L/min, 4.5 p.s.i., 110V, 50/60 Hz); después que las esferas estuvieron disueltas, los huevos fueron recuperados decantando el agua y posteriormente utilizando un microscopio estereoscópico para cuantificarlos. Para conservar la misma proporción de hembras por tratamiento, en aquellos tratamientos donde ocurrió la muerte de una hembra, ésta fue remplazada por otra. Las variables registradas en este estudio fueron la fecundidad, la cual se expreso como huevo/hembra/día y el porcentaje de hembras que ovipositaron sobre el substrato artificial con respecto al total de hembras colocadas en las jaulas.

Selección del Tipo de Sustrato Artificial de Oviposición. Este experimento consistió en la evaluación de seis tipos de telas que fueron cubiertas con una película transparente de silicón, las telas utilizadas fueron: organza color verde (Pantone156437), organza blanca, organza negra, sifón blanco, tuzor verde (Pantone16-6444) y tuzor blanco. Cada color y tipo de tela, fue considerado como un tratamiento, las cuales fueron colocadas en uno de los costados de las jaulas tipo Mission (Fig. 1) y constituyó el sustrato de oviposición ubicados en la cámara húmeda de la jaula. El

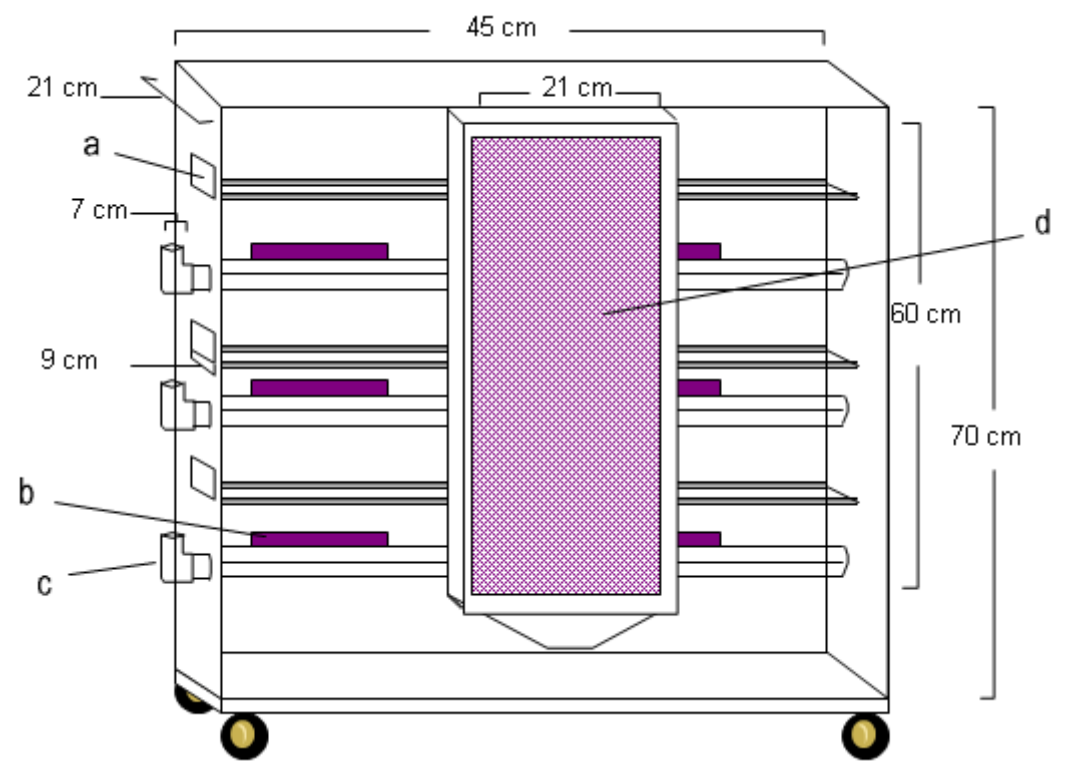

Figura 1. Jaula tipo Mission adaptada para la colonización de Anastrepha fraterculus. (a) Canaleta para colocar las pupas y el alimento. (b) Papel filtro para proporcionar el agua a las moscas. (c) Tubo de PVC para el agua. (d) Panel para oviposición y colecta de huevos. 
estudio consistió de seis tratamientos y cada uno con 10 repeticiones, cuyo diseño experimental fue completamente al azar. Las moscas evaluadas en este estudio correspondieron a la 6a. generación, las cuales fueron alimentadas con el tipo de alimento previamente descrito.

Para aumentar el área de reposo y reducir el estrés de las moscas, la parte inferior de las jaulas fue acondicionada con papel estraza corrugada. Un contenedor con la cantidad de 1,000 pupas de $24 \mathrm{~h}$ antes de emerger fue colocado en el interior de cada jaula. Diez días después de la emergencia, los adultos alcanzaron la maduración sexual, entonces se inició la colecta de huevos, actividad que se realizó durante 10 días consecutivos. Para mantener hidratado el huevo antes de las colectas, se aplicó una capa de fucelleron (elaborado con $300 \mathrm{~g}$ de fucelleron, 15 litros de agua y $3.12 \mathrm{~g}$ de detergente Timsen ${ }^{\circledR}$, hervido y enfriado por $24 \mathrm{~h}$ ) utilizando una bomba aspersora manual a una presión de $80 \mathrm{lb} / \mathrm{pulg}^{2}$. La aplicación sobre el panel se realizó en forma de abanico evitando que hubiera escurrimiento de fucelleron. La colecta de huevo se realizó de acuerdo a la metodología descrita para A. ludens y A. obliqua en condiciones de cría masiva (Domínguez et al. 2010). Las variables registradas en este estudio fueron el volumen de huevo colectado $(\mathrm{ml})$ y el porcentaje de eclosión del huevo.

Formulaciones de la Dieta Larvaria. La determinación de la dieta más eficaz para producir mayor cantidad de insectos se realizó sembrando las larvas recién eclosionadas en cinco diferentes dietas, que fueron formuladas de acuerdo con los ingredientes y proporciones requeridas para otras especies de moscas de la fruta indicadas en el Cuadro 1. Estas dietas son utilizadas para criar A. ludens (ALU), para A. obliqua (AOB), para C. capitata (CCA), dieta gélica de agar (DAG) dieta gélica de almidón (DAL) (Schwarz et al. 1985, Stevens 1991, Artiaga-López et al. 2004, Rivera et al. 2007, Domínguez et al. 2010). Cada tipo de dieta fue considerado un tratamiento y por cada tratamiento se realizaron 30 repeticiones, dando un total de 150 unidades experimentales, cuyo diseño experimental fue completamente al azar. Cada unidad experimental consistió en una caja de Petri de $100 \times 10 \mathrm{~mm}$ con 100 g de dieta. Cada unidad fue sembrada con 100 larvas recién eclosionadas. Los parámetros evaluados fueron: peso de larva (mg), supervivencia de huevo a larva (\%), pupación (\%) a las $24 \mathrm{~h}$, peso de pupa (mg), emergencia de adultos (\%), obtenidos de acuerdo a la metodología establecida para los laboratorios de cría de moscas de la fruta (Orozco et al. 1983, FAO/IAEA/USDA 2003, Hernández et al. 2005).

Análisis Generacional. Una vez determinada la dieta para el desarrollo de la larva y el panel para oviposición (tela tipo "organza” color blanco) se estableció una colonia de $A$. fraterculus y se determinó la fecundidad (huevo/hembra/día), supervivencia de huevo a larva (\%) y peso de larva (mg) durante 16 generaciones. La fecundidad fue determinada midiendo el volumen de huevo colectado por día por jaula durante 10 días de oviposición, la cuantificación del huevo se realizó considerando 16,000 huevos en un ml. La supervivencia de huevo a larva correspondió al porcentaje de 
Cuadro 1. Ingredientes y proporciones para elaborar $500 \mathrm{~g}$ de dieta para el desarrollo larvario de moscas de la fruta (según diferentes autores). (ALU = A. ludens; $\mathrm{AOB}=\mathrm{A}$. obliqua; $\mathrm{CCA}=\mathrm{C}$. capitata DAG = Gélica agar; DAL = Gélica almidón).

\begin{tabular}{|c|c|c|c|c|c|}
\hline Ingredientes & ALU & AOB & CCA & DAG & DAL \\
\hline Polvo de olote $(\mathrm{g})^{1}$ & 90 & 81.65 & - & - & - \\
\hline Harina de maíz (g) ${ }^{2}$ & 26.5 & 43.35 & - & 23.95 & - \\
\hline Bagazo de caña $(\mathrm{g})^{3}$ & - & - & 37.5 & - & - \\
\hline Salvado de trigo (g) ${ }^{4}$ & - & - & 20 & - & - \\
\hline Azúcar $(g)^{5}$ & 46 & 45 & 60 & 39.95 & - \\
\hline Levadura Lake-States (g) ${ }^{6}$ & 35 & 31.65 & 45 & 28 & - \\
\hline Levadura Coltec $(\mathrm{g})^{7}$ & - & - & - & - & 109.5 \\
\hline Acido Cítrico (g) ${ }^{8}$ & 2.2 & 2.2 & 12.5 & - & - \\
\hline Benzoato $(\mathrm{g})^{9}$ & 2 & 1 & 1.5 & 0.5 & 1.49 \\
\hline Nipagin (g) $)^{10}$ & 1 & 0.85 & 2 & 0.5 & 1 \\
\hline Goma guar $(\mathrm{ml})^{11}$ & 0.5 & 0.85 & - & - & - \\
\hline $\operatorname{Agar}(g)^{12}$ & - & - & - & 5.2 & - \\
\hline $\mathrm{HCl}(\mathrm{g})^{13}$ & - & - & - & 2.5 & 4.48 \\
\hline Agua (ml) & 296.8 & 291.6 & 284 & 399.4 & 383.5 \\
\hline
\end{tabular}

${ }^{1}$ Corn cob fractions 100, Mt. Pulaski Products Inc., Chicago IL.; ${ }^{2}$ Maíz Industrializado del Sureste, S.A. de C.V., Arriaga, Chiapas, México; ${ }^{4}$ Colpac, Navajoa, Sonora, México. ${ }^{5}$ Ingenio Huixtla, Chiapas, México; ${ }^{6}$ Lake States Div. Rhinelander Paper Co. Rhinelander Wis.; ${ }^{8}$ Anhidro acidulante FNEUM, Mexana, S.A. de C.V, Cuernavaca, Morelos, México. ${ }^{9} \mathrm{Cia}$. Universal de Industrias, S.A. de C.V., México, D.F; ${ }^{10}$ Mallinckrodt Speciality, Chemicals Co. St. Louis Miss.; ${ }^{11}$ Tic gums, Inc. Belcamp, Md.; ${ }^{13}$ Omnichem, Procesos Químicos Científicos, S.A. de C.V., Puebla, Puebla, México.

larvas recuperadas con respecto al número de huevos sembrados en la dieta larvaria. El peso de larva fue estimado con base en el número de larvas recuperadas en cada dieta, considerando el número de larvas en un mg y los gramos totales de larvas recuperadas. Las estimaciones fueron hechas de acuerdo a los métodos descritos por Hernández et al. (2005) y Rivera et al. (2007).

Supervivencia, Fecundidad y Expectativa de Vida. La colonia de A. fraterculus fue establecida y mantenida durante 16 generaciones utilizando la dieta utilizada para criar A. ludens para el desarrollo de la larva y el panel para oviposición de tela tipo organza color blanco. En la generación 16 se determinaron los parámetros de supervivencia, fecundidad y expectativa de vida, para ello se cuantificaron el número de huevos por hembra por día y el número de muertos por día, actividad que se realizó hasta que murió la última hembra. Además de elaborar las curvas de supervivencia y expectativa de vida, se estimaron los parámetros demográficos: tasa de reproducción neta $\left(\mathrm{R}_{\mathrm{o}}\right)$, tasa intrínseca de crecimiento poblacional $\left(\mathrm{r}_{\mathrm{m}}\right)$, tiempo medio de gene- 
ración $\left(\mathrm{T}_{\mathrm{G}}\right)$ y tiempo de duplicación de la población $\left(\mathrm{T}_{\mathrm{d}}\right)$ de acuerdo a los métodos descritos por Carey (1993). Los datos de supervivencia y fecundidad se obtuvieron realizando siete repeticiones. Cada repetición consistió en seleccionar 100 pupas y colocarlas en jaulas de vidrio de $30 \times 30 \times 30 \mathrm{~cm}$, que permanecieron a $80 \%$ de H.R. y un fotoperíodo de 12:12 horas luz: oscuridad. Los insectos fueron provistos de agua contenida en un frasco de vidrio de $500 \mathrm{ml}$ con una pieza de papel filtro y algodón, y alimento (azúcar: proteína hidrolizada, 3:1). Para la obtención de huevos se colocaron 10 esferas de fucelleron. La fecundidad fue determinada en base al número de huevos colectados por día por jaula, considerando el número de hembras vivas. La supervivencia se estimó en base al número de moscas muertas por día.

Análisis de Datos. La selección del color del sustrato para oviposición se determinó con base al número de huevos colectados, y posteriormente sometidos a un análisis de co-varianza, donde se consideró como factor la variación en el color de las esferas de fucelleron y el día de oviposición como co-variable. Previamente al análisis, se determinó mediante la prueba de Bartlett (Zar 1999) la existencia de homogeneidad en las varianzas $(\mathrm{P}<0.05)$. Posteriormente, debido a que sólo hubo diferencias en los valores de los colores de las esferas se realizó la separación de medias aplicando la prueba de Tukey $(P \leq 0.05)$.

Las dietas fueron evaluadas con base a los porcentajes de supervivencia de huevolarva, pupación a las 24 h y emergencia de adultos. Antes de realizar los análisis, los datos fueron transformados a grados del arco-seno en radianes de la raíz cuadrada de la proporción $\mathrm{x}^{\prime}=\operatorname{Sen}^{-1} \sqrt{ } \chi$, donde $\chi$ correspondió al valor original como proporción (porcentaje/100) (Underwood 2005). Con los datos así transformados se realizó un análisis de varianza (ANOVA) y la separación de medias se hizo de acuerdo a la prueba de Tukey $(P \leq 0.05)$ (SAS Institute 2003).

Los datos de los pesos de larva y pupa de todos los experimentos fueron sometidos a un ANOVA y la separación de medias se hizo de acuerdo a la prueba de Tukey $(P \leq 0.05)$ (SAS Institute 2003).

Los datos de los parámetros demográficos fueron analizados de acuerdo a método propuesto por Carey (1993).

\section{RESULTADOS}

Selección del Color del Sustrato de Oviposición. La fecundidad se expresó como huevos por hembra por día. El análisis de co-varianza indicó que el color de las esferas fue un factor que influyó en forma significativa sobre dicha variable $(F=11.26$; g.l. $=5$; 593; $P<0.001)$. Se observó que las hembras tuvieron preferencia por los sustratos de colores claros, específicamente por las esferas de color amarillo en donde se registró la mayor cantidad de huevos, mientras que en los sustratos de color negro hubo menor oviposición. Los sustratos de oviposición con valores intermedios fueron 
el color azul, rojo, transparente y verde, respectivamente (Cuadro 2). Sin embargo, el día de oviposición que fue considerado como una co-variable en el análisis reveló que su efecto sobre la fecundidad no fue significativo $(F=2.85$; g.l. $=1 ; 594 ; P=0.0919)$. Independientemente del color de esfera utilizado, la mayor producción de huevos se registro en el primer día, a los 4 y 8 días de oviposición (Fig. 2).

Selección del Sustrato Artificial de Oviposición. Para las evaluaciones de distintos paneles de oviposición, el panel elaborado con tela tipo organza de color blanco cubierto con una película de silicón transparente fue donde se obtuvo la mayor cantidad de huevos ( $0.3 \mathrm{ml} \sim 17$ huevos/hembra/día; Fig. 3a), mientras que la mayor eclosión de larvas se registró en los huevos colectados en el panel elaborado con tela organza color negra (58.3\%; Fig. 3b), a pesar que con este tipo de panel se colectaron $0.186 \mathrm{ml}$ de huevos (10 huevos/hembra/día).

En el panel hecho con tela tipo tuzor color blanco se obtuvo una colecta de $0.1 \mathrm{ml}$ de huevos que correspondió al menor volumen colectado; en dicho panel también se registró el menor porcentaje de eclosión larvaria (39.3\%; Fig. 3b). En ambos casos, las diferencias entre los valores observados fueron significativas (oviposición, $F=$ 57.74; g.l. = 5,354; $P<0.001$; eclosión larvaria, $F=15.81$; g.l. = 5, 354; $P<0.001$ ).

Formulaciones de la Dieta Larvaria. Los valores promedio obtenidos para las variables analizadas para cada tipo de dieta se muestran en el Cuadro 3. El mayor valor de supervivencia de huevo-larva se registró en la dieta ALU (26.9 $\pm 1.5 \%)$, mientras que el menor valor fue obtenido con la dieta DAL (2.17 $\pm 0.24 \%)$, las diferencias observadas entre las distintas dietas fueron significativas $(F=11.19$; g.l. $=$ 4, 102; $P<0.001$ ) (Cuadro 3). El peso de larva también mostró diferencias entre los tratamientos $(F=7.762$; g.l. $=4,102 ; P<0.001)$, donde el mayor valor que fue de $19.6 \mathrm{mg}$ correspondió a la dieta ALU, mientras que el menor valor fue, $14.5 \mathrm{mg}$ se registró para la dieta DAL. Los valores de peso de pupa tuvieron la misma tendencia que el peso de larva $(F=6.99 ;$ g.l. $=4,102 ; P<0.001)$. En cuanto al porcentaje de

Cuadro 2. Fecundidad de hembras de Anastrepha fraterculus utilizando esferas de fucelleron de diferentes colores como dispositivos de oviposición durante el proceso de colonización.

\begin{tabular}{cc}
\hline Color de esferas & Huevos/hembra/día \pm E.E.* \\
\hline Amarillo & $40.74 \pm 2.26 \mathrm{a}$ \\
Verde & $35.08 \pm 2.49 \mathrm{ab}$ \\
Transparente & $33.65 \pm 2.16 \mathrm{ab}$ \\
Azul & $29.59 \pm 2.66 \mathrm{~b}$ \\
Rojo & $28.06 \pm 2.31 \mathrm{~b}$ \\
Negro & $18.59 \pm 1.68 \mathrm{c}$ \\
\hline
\end{tabular}

* Valores con la misma letra en las columnas indica que no hay diferencia significativa $(\alpha=0.05)$. 
Flores et al. Sistema de cría artificial para A. fraterculus

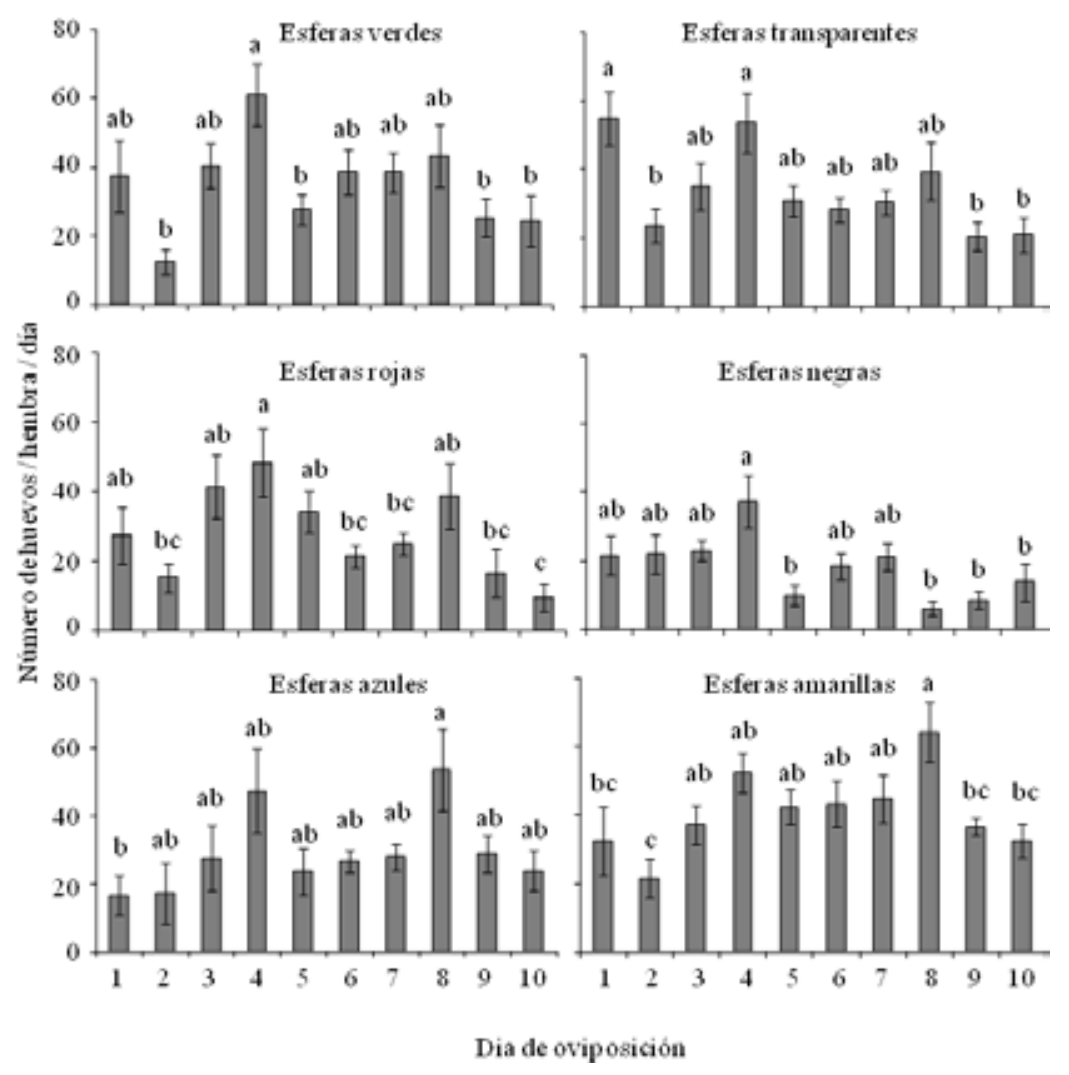

Figura 2. Cantidad de huevos ovipositados por hembras de Anastrepha fraterculus en esferas de fucelleron de diferentes colores. $N=10$ jaulas/color, cada jaula con cinco hembras y cinco machos.

Cuadro 3. Desarrollo larvario y parámetros de calidad observados en Anastrepha fraterculus con diferentes dietas.

\begin{tabular}{ccccccc}
\hline $\begin{array}{c}\text { Tipo de } \\
\text { dieta }\end{array}$ & $\begin{array}{c}\text { Supervivencia de } \\
\text { huevo-larva (\%) }\end{array}$ & $\begin{array}{c}\text { Peso de } \\
\text { larva (mg) }\end{array}$ & $\begin{array}{c}\text { Pupación a } \\
24 \mathrm{~h}(\%)\end{array}$ & $\begin{array}{c}\text { Peso de } \\
\text { pupa (mg) }\end{array}$ & $\begin{array}{c}\text { Emergencia } \\
(\%)\end{array}$ & $N^{*}$ \\
\hline ALU & $26.9 \pm 1.5 \mathrm{a}$ & $19.6 \pm 0.1 \mathrm{a}$ & $97.7 \pm 0.8 \mathrm{a}$ & $16.7 \pm 0.2 \mathrm{a}$ & $83.3 \pm 1.4 \mathrm{a}$ & 18 \\
AOB & $15.8 \pm 1.6 \mathrm{bc}$ & $18.0 \pm 0.5 \mathrm{a}$ & $90.1 \pm 4.5 \mathrm{a}$ & $14.2 \pm 0.5 \mathrm{ab}$ & $79.3 \pm 5.4 \mathrm{a}$ & 19 \\
CCA & $13.7 \pm 1.2 \mathrm{c}$ & $17.9 \pm 0.4 \mathrm{a}$ & $98.4 \pm 3.2 \mathrm{a}$ & $12.6 \pm 0.9 \mathrm{~b}$ & $49.3 \pm 7.8 \mathrm{~b}$ & 29 \\
DAG & $23.6 \pm 3.7 \mathrm{ab}$ & $18.6 \pm 0.5 \mathrm{a}$ & $94.6 \pm 2.1 \mathrm{a}$ & $13.5 \pm 0.5 \mathrm{~b}$ & $30.1 \pm 5.6 \mathrm{~b}$ & 29 \\
DAL & $2.2 \pm 0.2 \mathrm{~d}$ & $14.5 \pm 1.2 \mathrm{~b}$ & $100 \pm 0.0 \mathrm{a}$ & $11.1 \pm 0.6 \mathrm{~b}$ & $59.6 \pm 12.2 \mathrm{ab}$ & 12 \\
\hline
\end{tabular}

ALU (Stevens, 1991), AOB (Artiaga-López et al., 2004), CCA (Schwarz et al., 1985). DAG, DAL (Rivera et al., 2007).

* Número de repeticiones. Valores con la misma letra en las columnas indica que no hay diferencia significativa ( $\alpha$ $=0.05$ ). 

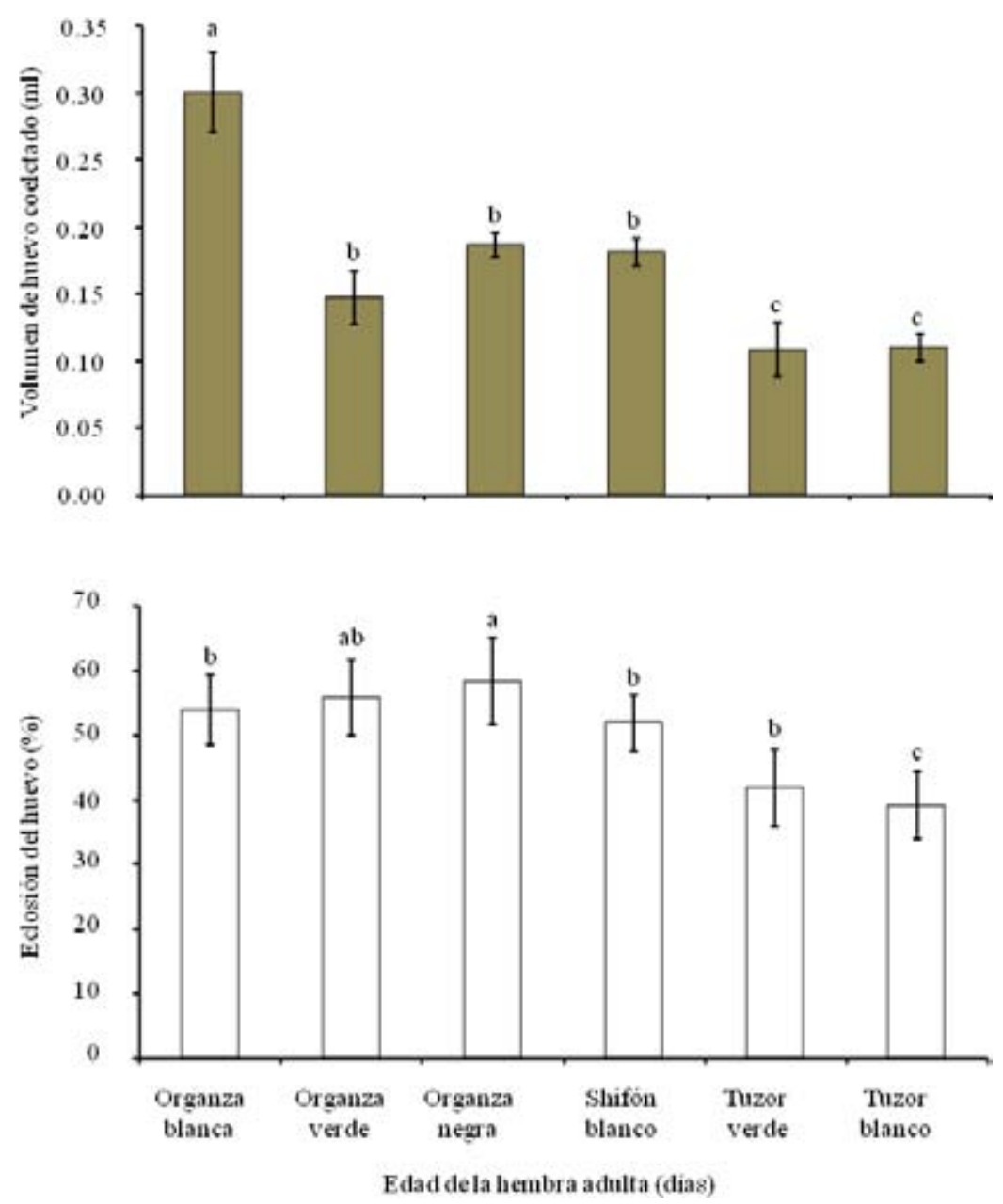

Figura 3. Cantidad de huevos (mL) ovipositados por hembras y porcentaje de huevo eclosionado de Anatrepha fraterculus en jaulas tipo Missión con paneles de diferentes colores y textura.

pupación a las 24 horas no se observó diferencias significativas entre los tratamientos $(F=2.62$; g.l. $=4,102 ; P=0.059)$. El mayor porcentaje de emergencia (83.33 \pm $1.40 \%)$ se registró en las pupas que se desarrollaron en la dieta ALU, y el menor valor (30.10 $\pm 5.57 \%$ ) se observó en las pupas recuperadas de la dieta DAG, las diferencias fueron significativas $(F=10.84$; g.l. $=4,102 ; P<0.001)$.

Supervivencia, Fecundidad y Expectativa de Vida. La curva de supervivencia descrita por el número de individuos vivos por edad (x) indicó que la mortalidad de esta especie sucedió en forma gradual, durante los primeros 30 días. Si bien en los 
siguientes 30 días la mortalidad se incrementó, aquellos individuos que alcanzaron los 60 días de edad, vivieron en total 90 días (Fig. 4).

El número de moscas vivas, expresado como número $\left(\mathrm{n}_{\mathrm{x}}\right)$ o proporción $\left(\mathrm{l}_{\mathrm{x}}\right)$, indicó que el 50\% de la población inicial vivió 40 días. Mientras que la expectativa de vida al momento de la emergencia de los adultos correspondió a 38.74 días, la cual disminuyó en forma gradual, registrando un ligero incremento entre los 60 y 70 días de edad (Fig. 4).

Las moscas que alcanzaron 40 días de edad tuvieron una expectativa de vida promedio de 20 días más por vivir, y las que sobreviven, pueden vivir 20 días más. De tal manera que las moscas que llegan a esa edad tienen mayor probabilidad de alcanzar la siguiente edad.
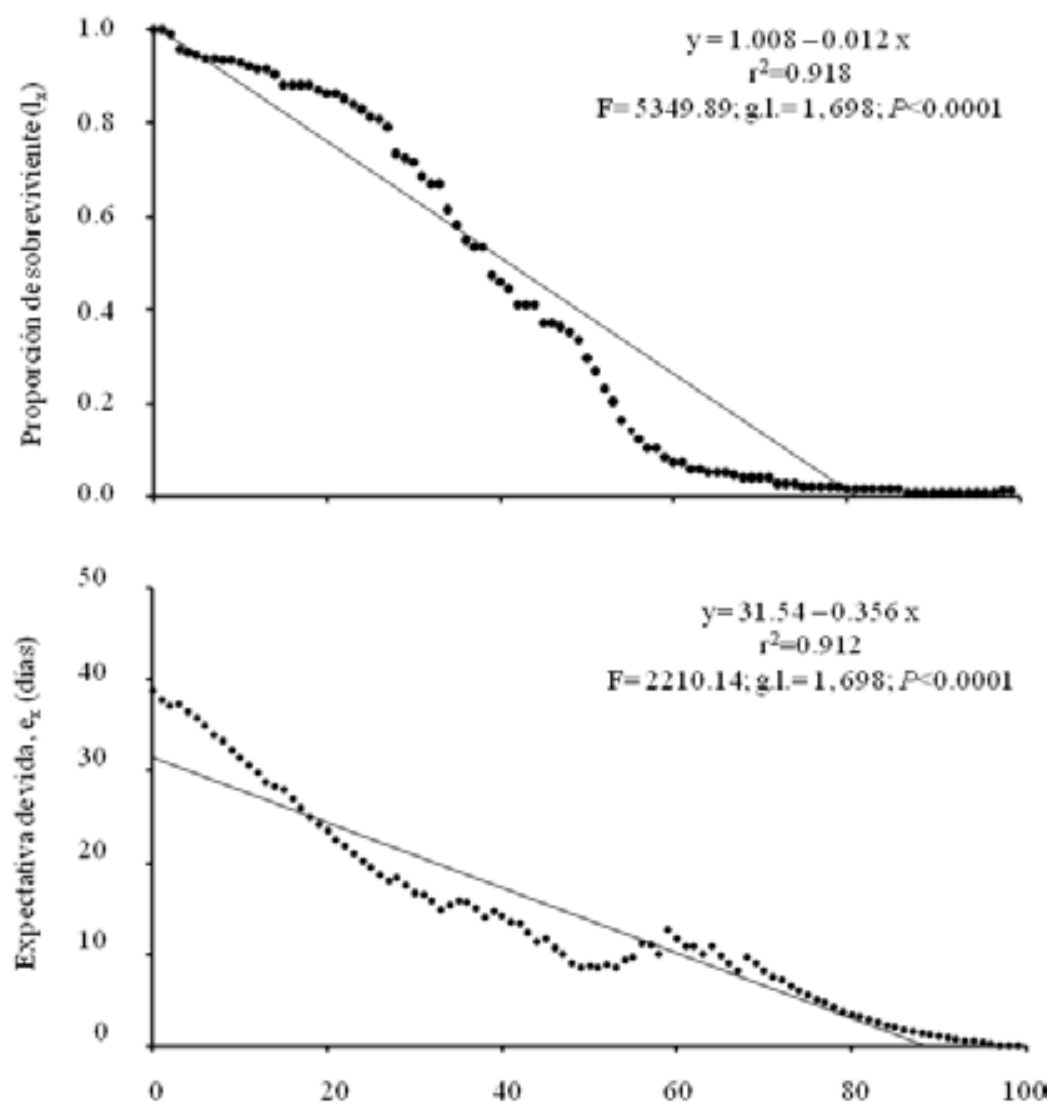

Edad de la hembra adulta (dias)

Figura 4. Proporción de sobrevivientes $\left(\mathrm{l}_{\mathrm{x}}\right)$ y expectativa de vida $\left(\mathrm{e}_{\mathrm{x}}\right)$ de Anastrepha fraterculus. 
Las hembras de $A$. fratereculus, empezaron a ovipositar a los ocho días de edad, continuaron haciéndolo hasta los 68 días, por lo que el período de oviposición fue de 60 días (Fig. 5).

El número de hijas/hembra/día en promedio durante toda la vida de las hembras correspondió a $345.38 \pm 44.5$. Mientras que el máximo número de huevos por hembra por día fue de 33 en la generación 12 (Fig. 6).

La supervivencia de huevo-larva sufrió variación durante las 16 generaciones en un intervalo de 10-30\%, la supervivencia de larva-pupa se mantuvo constante, solo en la $G_{12}$ disminuyó a $63 \%$, pero en la siguiente generación se incrementó y se mantuvo hasta la $\mathrm{G}_{16}$ en un intervalo de $80-98 \%$.

La tasa de reproducción neta (Ro) fue de $345.38 \pm 44.5$, la tasa intrínseca de crecimiento de (rm) $0.35 \pm 0.02$, la tasa finita de crecimiento $(\lambda) 1.42 \pm 0.03$ y el tiempo de duplicación de la generación (td) fue de $20.2 \pm 0.12$ días (Cuadro 4).

Durante las 16 generaciones, los pesos de larva y de pupa permanecieron sin variaciones significativas registrándose de 16 a $18 \mathrm{mg}$, y de 12 a 14 mg, respectivamente (Fig. 7).

\section{DISCUSIÓN}

Las hembras de A. fraterculus tuvieron preferencia por ovipositar en sustratos de colores claros y en esferas de color amarillo. Esta respuesta observada coincidió con la que se ha registrado en $A$. striata, la cual también tiene preferencia por ovipositar

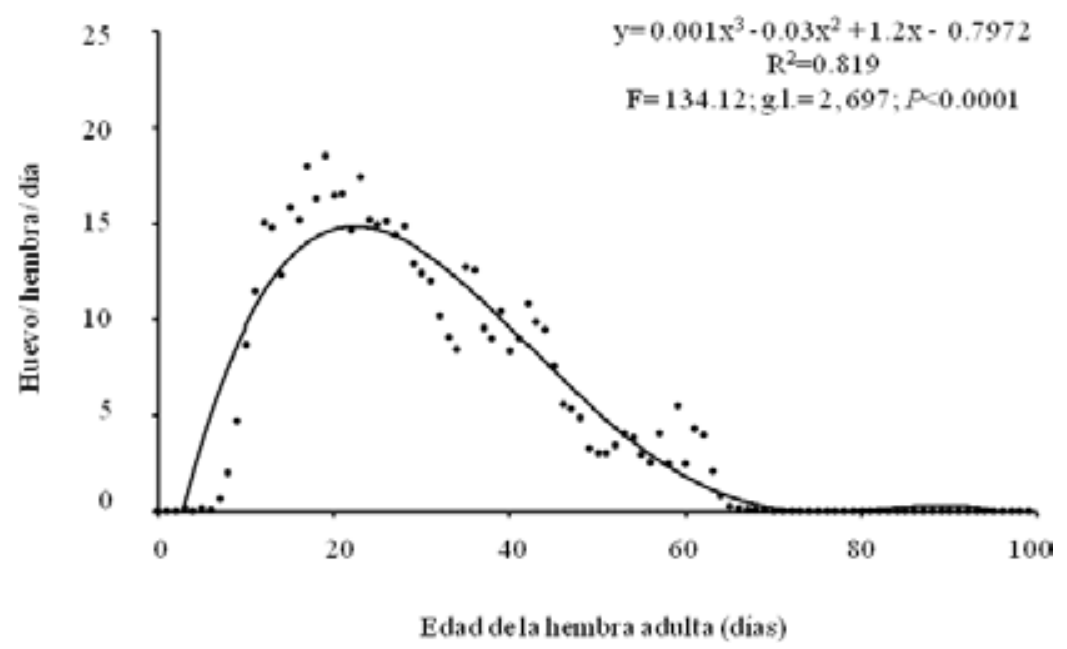

Figura 5. Fecundidad diaria de Anastrepha fraterculus utilizando tela tipo organza de color blanco cubierta con una capa de silicón transparente como sustrato de oviposición. 
Flores et al. Sistema de cría artificial para A. fraterculus

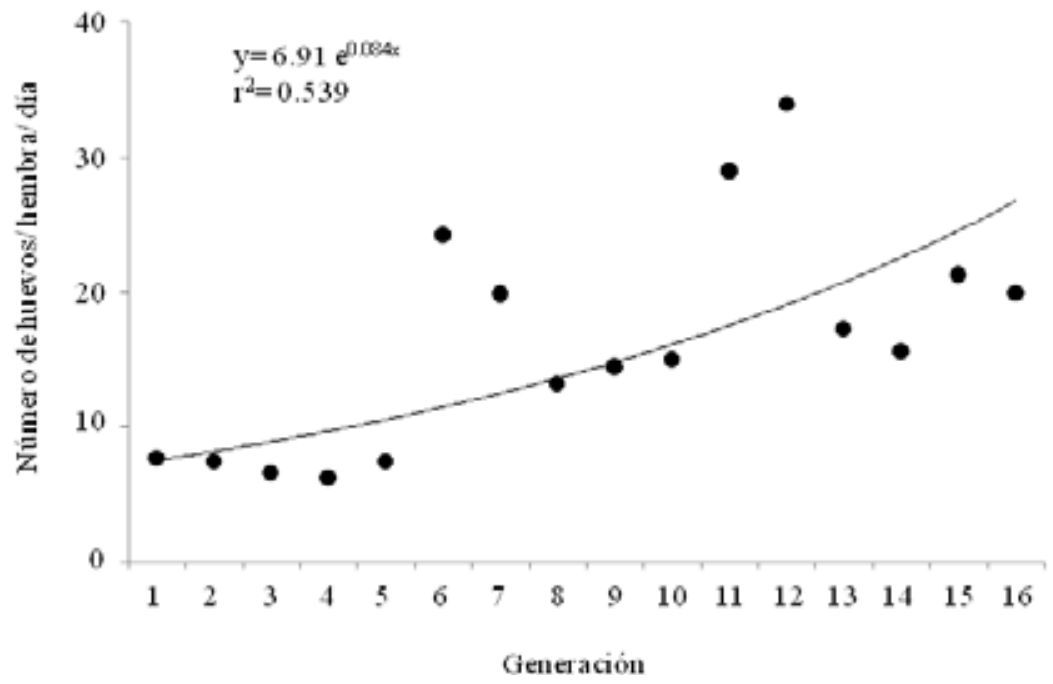

Figura 6. Fecundidad de $A$. fraterculus criadas en condiciones artificiales durante 16 generaciones bajo condiciones ambientales controladas.

Cuadro 4. Parámetros demográficos observados en adultos de Anastrepha fraterculus en la generación 16 durante el proceso de colonización.

\begin{tabular}{lc}
\hline \multicolumn{1}{c}{ Parámetros demográficos } & Promedio \pm E.E. \\
\hline Tasa de reproducción neta $\left(\mathrm{R}_{\mathrm{o}}\right)$ & $345.38 \pm 44.5$ \\
Tasa intrínseca de crecimiento $\left(\mathrm{r}_{\mathrm{m}}\right)$ & $0.35 \pm 0.02$ \\
Tiempo medio de generación $\left(\mathrm{T}_{\mathrm{G}}\right)$ & $16.82 \pm 0.82$ \\
Tiempo de duplicación de la población $\left(\mathrm{T}_{\mathrm{d}}\right)$ & $20.2 \pm 0.12$ \\
\hline
\end{tabular}

en esferas de color verde y azul claro (Hernández et al. 2004). La misma tendencia de oviposición fue observada en $A$. fraterculus al tener preferencia por ovipositar en paneles planos elaborados con tela tipo organza de color blanco recubiertos con una capa de silicón transparente. Este tipo de paneles son utilizados para la oviposición de Anastrepha obliqua (Artiaga-López et al. 2004), mientras que las hembras de A. striata tienden a ovipositar mayor cantidad de huevos en paneles tridimensionales elaborados con domos de mallas plásticas recubiertas con silicón teñidos de color negro e insertados en una placa de acrílico trasparente (Hernández et al. 2004). Las hembras de A. ludens ovipositan mayor cantidad de huevos sobre paneles de tela tipo lino catalán color negro cubierto con una capa de silicón. Pero las hembras de Ceratitis capitata tienen preferencia por ovipositar a través de una malla tull (Malla 57) y las de A. serpentina en tela tipo bombay de color azul. Para que las hembras de A. fra- 

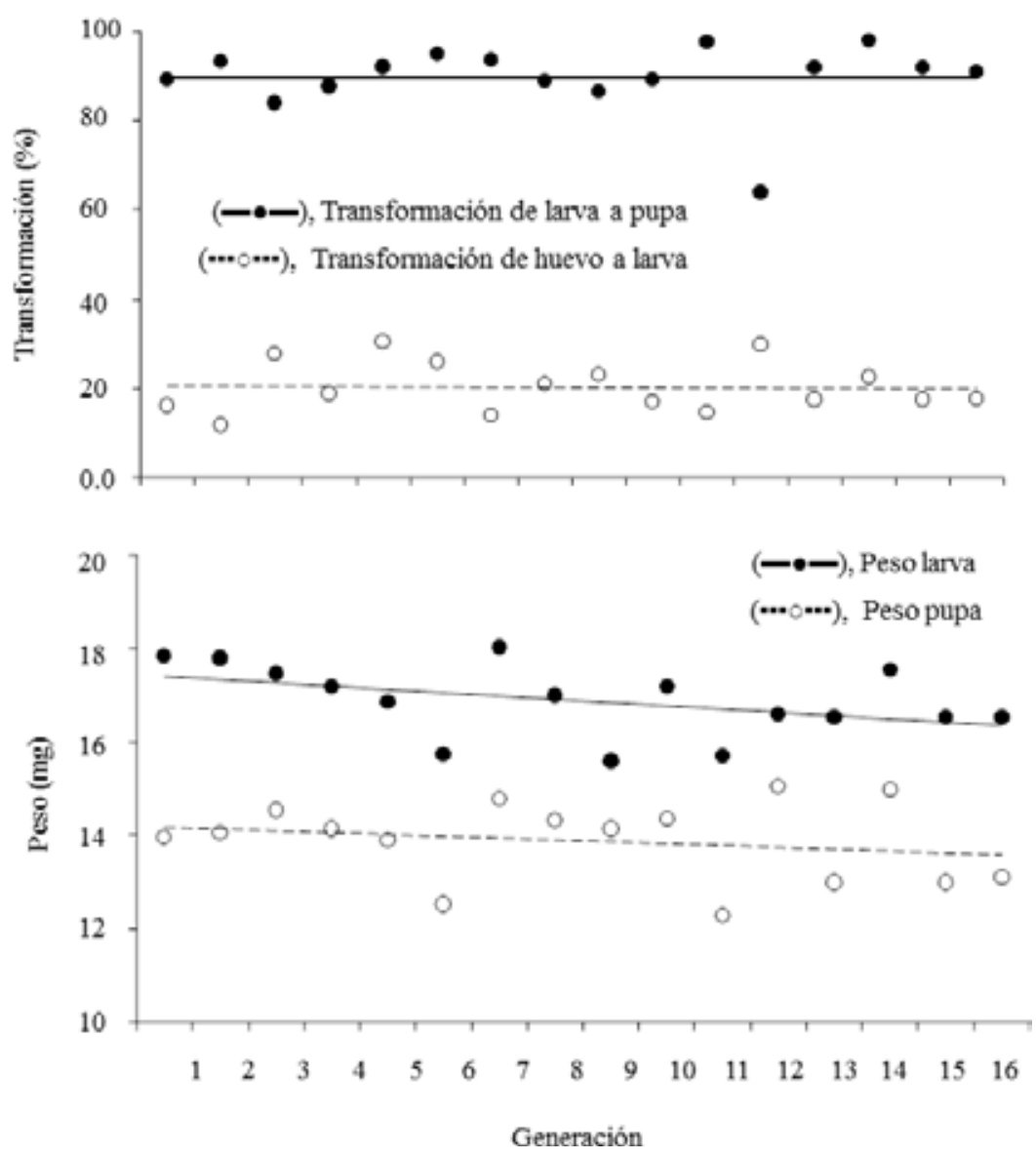

Figura 7. Calidad de larvas y de pupas de A. fraterculus criadas en condiciones ambientales controladas durante 16 generaciones.

terculus ovipositen, se han utilizado como dispositivos de oviposición esferas de agar tratadas con jugo de fruta de guayaba antes de envolverlas con membranas de papel parafilm (Salles 1992), domos de parafina teñidos de color y sustratos elaborados con tela nylon de color rojo (Jaldo et al. 2007, Alama 1999). También se ha logrado que ovipositen en sustratos elaborados con tela de color blanco recubiertos con una película de silicón (Núñez-Bueno \& Guzmán-Dueñas 1999, Jaldo et al. 2001). Estos resultados indican que se trata de una especie con mucha flexibilidad para adaptarse a ovipositar en diferentes dispositivos de oviposición. La selección de un sustrato está basada en la preferencia que tiene una especie por un determinado color. De esta forma, se ha observado que las moscas tienen preferencia por colores que producen 
contraste, así se determino que para $A$. ludens el sustrato fue de color negro (Stevens 1991), para A. serpentina de color azul (Pinson et al. 2006), para C. capitata y A. obliqua de color blanco (Schwarz et al. 1985, Artiaga-López et al. 2004). En este estudio A. fraterculus tuvo preferencia para ovipositar en esferas de color amarillo que se identificaron como un color claro, pero también ovipositó en esferas de color verde identificadas como oscuras, en ambos casos el color de las esferas contrasta con el ambiente, este hecho indicó que las moscas logran identificarlas como un recurso para oviposición. Independiente del color de las esferas se pudieron colectar al menos 18 huevos por hembra por día, al igual que en el panel elaborado con tela organza de color blanco donde se obtuvieron 17 huevos/hembra/día. En ambos casos, el número de huevos fue ligeramente mayor a los 15 huevos/hembra por colecta que se han reportado en otros estudios con A. fraterculus utilizando panel (Vera et al. 2007).

El tamaño normal de las larvas para dar origen a adultos de buena calidad depende de la calidad nutritiva de la dieta utilizada durante el desarrollo. En este estudio, las larvas de $A$. fraterculus registraron un mayor desarrollo con la dieta elaborada con polvo de olote, ingrediente utilizado para las dietas de A. ludens, A. obliqua, y A. serpentina (Artiaga-López et al. 2004, Stevens 1991, Pinson et al. 2006). Este hecho indicó que las especies del género Anastrepha tienen un mayor aprovechamiento de los nutrientes a partir de las dietas que poseen polvo de olote. Inicialmente para el desarrollo larvario de $A$. fraterculus se utilizó una dieta larvaria a base de levadura torula y polvo de zanahoria, posteriormente se utilizó una dieta a base de germen de trigo y levadura de cerveza en donde se obtuvieron rendimientos larvarios con valores intermedios (González et al. 1971, Jaldo et al. 2001, Salles 1992). Al sustituirse el polvo de zanahoria por germen de trigo, se formuló una dieta con mejores características físico-químicas que se reflejó en un incremento en la producción de insectos con mayor calidad (Núñez-Bueno \& Guzmán-Dueñas 1999). La dieta que contenía 7\% de proteína indujo a tener una mayor recuperación larvaria; sin embargo, autores como Braga-Sobrinho et al. (2010), recomienda una dieta con 9\% de proteína para esta especie. Por otra parte, en este trabajo se obtuvo una supervivencia de huevo a larva del 27\%; mientras que Vera et al. 2007 obtuvo un 66\% cuando utilizó el bagazo de caña como agente texturizante.

La supervivencia que registraron los adultos de $A$. fraterculus indicó que la mortalidad ocurrió en forma gradual hasta una edad de 60 días. En el caso de adultos de $A$. ludens se ha observado que el $50 \%$ de individuos viven entre 25 y 36 días, mientras que los adultos de $A$. obliqua viven entre 51 a 62 días, y los adultos de $A$. serpentina de 46 a 55 días. Este hecho indicó que los adultos de $A$. fraterculus tienen una mayor longevidad en condiciones de laboratorio en comparación con adultos de otras especies del género Anastrepha (Celedonio-Hurtado et al. 1988).

La expectativa de vida de $A$. fraterculus fue de 38.74 días, a diferencia de $A$. ludens que es de 110.7 días, para A. obliqua de 79.2 días, en $A$. serpentina de 75.5 días 
y para A. striata de 61.4 días (Liedo et al. 1993). Sin embargo, Jaldo et al. (2007) obtuvieron curvas de supervivencia que indican que el $70 \%$ de los machos alcanzan una edad de 88 días y el 50\% de las hembras al menos viven 64 días. Este hecho indicó que los parámetros demográficos que describen una población de laboratorio difiere dependiendo del origen geográfico y deben ser considerados para establecer los parámetros de calidad de una cría sostenible de insectos.

Con respeto a la fecundidad, las hembras de $A$. fraterculus registraron su mayor producción de huevos entre los 15 y 22 días de edad, con una oviposión aproximada de 11 huevos/hembra/día, que es menor a 19.75 huevos reportado por Salles (1993), pero fue mayor a 2.5 y 0.6 huevos/hembra/día que se registró en moscas que se desarrollaron en guayaba y manzana (Malus domestica L.), respectivamente (Sugayama et al. 1998). Adicionalmente, la fecundidad de esta especie es ligeramente mayor a los 9 huevos/hembra/día que se estimó en A. obliqua (Hernández et al. 2009), de 10.79 en $A$. ludens y de 6.46 en $A$. serpentina y fue menor a 14.95 huevos/hembra/día reportado en $A$. obliqua (Liedo et al. 1992).

La tasa neta de reproducción de A. fraterculus fue menor a la observada en $A$. ludens y mayor a la de A. obliqua y de A. serpentina (Liedo et al. 1993). Esto sugiere que $A$. fraterculus tiene una alta capacidad reproductiva indicando que es factible su establecimiento a un sistema de cría masiva bajo condiciones ambientales controladas. Lo anterior se confirmó con el alto valor de la tasa intrínseca de crecimiento poblacional y la tasa finita. Por tal motivo se considera que el tiempo de duplicación de la población de $A$. fraterculus es mayor que al observado en A. ludens, A. obliqua, A. serpentina y A. striata.

Con respecto a los pesos de larva y de pupa, observados en $A$. fraterculus durante 16 generaciones, estos fueron similares a los establecidos en la cría masiva de $A$. obliqua y A. ludens (Artiaga-López et al. 2004). El peso de pupa obtenido en este trabajo con la dieta elaborada a base de levadura torula y polvo de olote permitió obtener pupas con un peso mayor a $14 \mathrm{mg}$, Este valor corresponde a 1-mg más de peso que las obtenidas por Vera et al. (2007) con dieta a base de levadura torula y bagazo de caña, pero fue de menor peso comparadas con las pupas de $18.43 \mathrm{mg}$ obtenidas por BragaSobrinho et al. (2006) con dieta preparada a base de bagazo de caña y levadura de cerveza. La supervivencia de huevo a larva observada (27\%) fue menor a lo reportado (66\%) por Vera et al. (2007), pero fue mayor a lo reportado por otros investigadores para esta misma especie (Núñez-Bueno \& Guzmán-Dueñas 1999, Alama 1999).

En conclusión, considerando los valores de los parámetros medidos en los insectos criados, la adaptación de A. fraterculus a un sistema artificial fue el resultado de la interacción de varios factores durante el proceso de colonización y cría masiva, así como la capacidad genética de adaptación inherente a esta especie, a través de las generaciones observadas. A pesar de que los resultados obtenidos en los parámetros de calidad cumplen en forma parcial con los estándares establecidos en los laborato- 
rios de cría de moscas de la fruta (Orozco et al. 1983, FAO/IAEA/USDA 2003), la información generada garantiza el establecimiento de su cría artificial y es factible mejorar dicho sistema para alcanzar niveles masivos que cumplan con los estándares de calidad y lograr la aplicación de la TIE para el manejo dicha plaga en el área que se considere pertinente.

Agradecimientos. A Liliana López (CNCMF), por la confirmación de la especie. A Ezequiel de León (ECOSUR), por su apoyo en la colecta de frutos. A la Subdirección de Desarrollo de Métodos del Programa Moscamed-Moscafrut (SAGARPA-IICA), por las facilidades proporcionadas para realizar esta investigación.

\section{LITERATURA CITADA}

Alama, D. 1999. Ensayos para determinar niveles de oviposición de Anastrepha fraterculus (Wied.) sobre fruta y dispositivos artificiales. Pp. 79-83. En: The South American fruit fly, Anastrepha fraterculus (Wied.). Advances in artificial rearing, taxonomic status and biological studies. International Atomic Energy Agency, IAEA Tech-Doc 1064. Austria.

Artiaga-López, T., E. Hernández, J. Domínguez-Gordillo, D. S. Moreno \& D. Orozco-Dávila. 2004. Mass-production of Anastrepha obliqua at the Moscafrut Fruit Fly Facility, Mexico. Pp. 389-392. In: B. N. Barnes [Ed.]. Proceedings of the $6^{\text {th }}$. International Symposium on Fruit Flies of Economic Importance. Isteg Scientific Publications Irene, South Africa.

Boller, E. 1968. An artificial oviposition device for the European cherry fruit fly, Rhagoletis cerasi. Journal of Economic Entomology, 61: 850-852.

Boller, E. F. \& D. L. Chambers. 1977. Quality aspects of mass-reared insects. Pp. 219-235. In: Ridway, R. L. and Vinson S. B. [Eds.]. Biological Control by Augmentation of Natural Enemies. Plenum Press. New York, USA.

Braga-Sobrinho, R., C. Caceres, A. Islam, V. Wornoayporn \& W. Enkerlin. 2006. Improving mass rearing technology for South American fruit fly (Diptera: Tephritidae). Caatinga, 19: 310-316.

Braga-Sobrinho, R., J.A. Guimaraes, E.L. Araujo \& M.A.B. Moreira. 2010. Suitable larval diets for Anastrepha fraterculus (Diptera: Tephritidae). Pp. 222. In: Sabater Muñoz, B., V. Navarro Llopis and A. Urbaneja [Eds.]. Book of abstracts of the $8^{\text {th }}$ International Symposium on Fruit Flies of Economic Importance. Universitat Politécnica de Valencia, Valencia, España.

Carey, J. R. 1993. Applied demography for biologists with special emphasis on insects. Oxford University Press. New York, USA.

Celedonio-Hurtado, H., M. Aluja, \& P. Liedo. 1995. Adult population fluctuations of Anastrepha species (Diptera: Tephitidae) in tropical orchard habitats of Chiapas, México. Environmental Entomology, 24: 861-869.

Celedonio-Hurtado, H., P. Liedo, M. Aluja, J. Guillen, D. Berrrigan \& J. Carey. 1988. Demography of A. ludens, A. obliqua and A. serpentina (Diptera: Tephritidae) in México. Florida Entomologist, 71: $111-120$.

Dadd, H. R. 1973. Insect nutrition: Current developments and metabolic implications. University of California at Berkeley, Cal. USA.

Domínguez, J., T. Artiaga-López, E. Solís \& E. Hernández. 2010. Métodos de colonización y cría masiva. Pp. 259-276. En: Montoya, P., J. Toledo y E. Hernández. [Eds.]. Moscas de la Fruta: Fundamentos y Procedimientos para su Manejo. S y G Editores. México, D. F. 
Eiseman, L. \& L. Herbert. 1990. The pantone book of de color. Over 1000 color standards, color basics and Guidelines for design, fashion, furnishings and more. Harry N. Abrams Inc., Publishers. New York, USA, 160 pp.

FAO/IAEA/USDA. 2003. Manual for product quality control and shipping procedures for sterile massreared tephritid fruit files. Version 5.0. Internacional Atomic Energy Agency. Viena, Austria.

Fontellas, T.M.L. \& F.S. Zucoloto. 2003. Effect of sucrose ingestión on the performance of wild Anastrepha obliqua (Macquart) females (Diptera:Tephritidae). Neotropical Entomology, 30:209-216.

González, J., C. Vargas \& B. Jara. 1971. Estudios sobre la aplicación de la técnica de machos estériles en el control de la mosca sudamericana de la fruta, Anastrepha fraterculus (Wied.). Revista Peruana de Entomología, 14: 66-86.

Hernández, E., T. Artiaga \& S. Flores. 2004. Development of an artificial oviposition device for Anastrepha striata Schiner (Diptera: Tephritidae). Pp. 393-398. In: B. N. Brian [Ed.]. Proceedings of the 6th International Symposium on Fruit Flies of Economic Importance. Isteg Scientific Publications, Irene, South Africa.

Hernández, E., S. Flores-Breceda, M. de la L. Sosa-Iturbe \& H. Ezquivel. 2005. Tamaño de unidad muestral y número de repeticiones para la estimación de los parámetros de desarrollo de Anastrepha obliqua y Anastrepha ludens (Diptera: Tephritidae). Folia Entomológica Mexicana, 44: 155-164.

Hernández, E., J. Toledo, T. Artiaga-López \& S. Flores. 2009. Demographic changes in Anastrepha obliqua (Diptera:Tephritidae) throughout the laboratory colonization process. Journal of Economic Entomology, 102: 542-551.

Jaldo, H. E., M. C. Gramajo \& E. Willink. 2001. Mass rearing of Anastrepha fraterculus (Díptera: Tephritidae): A preliminary strategy. Florida Entomologist, 84: 716-718.

Jaldo, H. E., E. Willink \& P. Liedo. 2007. Demographic analysis of mass-reared Anastrepha fraterculus (Diptera: Tephritidae) in Tucumán, Argentina. Revista Industrial y Agrícola de Tucumán, 84: 15-20.

Leppla, N.C, M. D, Huettel, D. L, Chambers, T. R; Ashley, D. H; Miyashita, T. T.Y. Wong \& E. J. Harris. 1983. Strategies for colonization and maintenance of the Mediterranean fruit fly. Entomologia Experimentalis et Applicata, 33: 89-96.

Liedo, P., J. R. Carey, H. Celedonio \& J. Guillen. 1992. Size specific demography of three species of Anastrepha fruit flies. Entomologia Experimentalis et Applicata, 63: 135-142.

Liedo, P., J. R. Carey, H. Celedonio \& J. Guillen. 1993. Demography of Anastrepha fruit flies: A case study of tree species of economic importance. Pp. 119-124. In: M. Aluja and P. Liedo [Eds.]. Fruit flies: Biology and Management. Springer-Verlag. New York, USA.

Message, C.M., \& F.S. Zucoloto. 1989. Effect of some artificial diets on egg production by Anastrepha obliqua. Revista Brasileira de Biología, 49: 699-701.

McPhail, M. \& F.E. Guiza. 1956. An oviposition medium for the Mexican fruit fly. Journal of Economic Entomology, 49:570.

Núñez-Bueno, L. \& R. Guzmán-Dueñas. 1999. Avances sobre la cría artificial de Anastrepha fraterculus (Wied.) (Díptera: Tephritidae) en Colombia. Informe Técnico. Instituto Colombiano Agropecuario-ICA. Ibagué, Tolima, Colombia.

Orozco-Dávila, D., A. Schwarz-Gehrke \& A. Pérez-Romero. 1983. Manual de procedimientos de control de calidad. Programa Mosca del Mediterráneo. Secretaría de Agricultura y Recursos Hidráulicos. México, D.F. 139 pp.

Ortiz, G. 1999. Potential use of the sterile insect technique against the South American fruit fly. Pp. 121-130. In: The South American fruit fly, Anastrepha fraterculus (Wied.): Advances in artificial rearing, taxonomic status and biological studies. International Atomic Energy Agency, IAEA TechDoc 1064. Austria. 
Pinson, E. P., L. O. Tejada, J. Toledo, W. Enkerlin, H. Celedonio-Hurtado, J. Valle, J. N. Pérez \& P. Liedo. 2006. Caracterización de la adaptación de Anastrepha serpentina (Wied.) (Diptera: Tephritidae) a condiciones de cría masiva. Folia Entomológica Mexicana, 45: 97-112.

Prokopy, R.J. 1966. Artificial oviposition devices for apple maggot. Journal of Economic Entomology, 59:231-232.

Prokopy, R.J. \& E. Boller. 1970. Artificial egging system for the European cherry fruit fly. Journal of Economic Entomology, 63:1414-1417.

Rivera, J.P, E. Hernández, J. Toledo, M. Salvador \& R. Silva. 2007. Dieta texturizada con agar para el desarrollo larvario de tres especies de moscas de la fruta (Diptera: Tephritidae). Folia Entomológica Mexicana, 46: 37-52.

Rössler, Y. 1975. Reproductive differences between laboratory reared and field collected populations of the Mediterranean fruit fly, Ceratitis capitata (Wied.). Annals of Entomological Society of America, 68: 987-991.

SAS Institute. 2003. JMP Statistical, Discovery Software. Version 5.0.1. SAS Institute Inc., Carg., North Carolina.

Salles L., A. B. 1992. Metodologia de criac de Anastrepha fraterculus (Wied., 1830) (Diptera: Tephritedae) em dieta artificial em laboratorio. Annais da Sociedade Entomológica do Brasil, 21: 479-487.

Salles L., A. B. 1993. Efeito da temperatura constante na oviposicao e no ciclo de vida de Anastrepha fraterculus (Wied. 1830) (Diptera: Tephritedae). Annais da Sociedade Entomológica do Brasil, 21: 58-62.

Schwarz, A. J., A. Zambada, D. H. S. Orozco, J. L. Zavala \& C. O. Calkins. 1985. Mass production of the Mediterranean fruit fly at Metapa, Mexico. Florida Entomologist, 68: 467-477.

Singh, P. 1984. Insect Diets; Historical Development, Recent Advances, and Future Prospects. Pp. 3244. In: King, E.G. and N.C. Leppla (Eds.). Advances and Challenges in Insect Rearing. Agricultural Research Service (Southern Region). U.S. Department of Agriculture, New Orleans.

Stevens, L. 1991. Manual of Standard Operating Procedures (SOP) for the Mass-rearing and Sterilization of the Mexican Fruit Fly, Anastrepha ludens (Loew). USDA-APHIS, South Central Region, Míssion, Texas, USA. 39 p.

Sugayama, R.L., A. Kovaleski, P. Liedo \& A. Malavasi. 1998. Colonization of a new fruit crop by Anastrepha fraterculus (Diptera: Tephritidae) in Brazil: A demographic analysis. Environmental Entomology, 27: 624-648.

Tanaka, N. 1965. Artificial egging receptacles for three species of tephritid flies. Journal of Economic Entomology, 58:177-178.

Underwood A., J. 2005. Experiments in ecology. Their logical design and interpretation using analysis. Cambridge University Press. 504 p.

Vargas, R.I., S. Mitchell, C. Hsu, \& W.A. Walsh. 1994. Laboratory evaluation of diets of processed corncob, torula, yeast, and wheat germ on four developmental stages of Mediterranean fruit fly (Diptera:Tephritidae). Journal of Economic Entomology, 87: 91-95.

Vera, M. T., S. Abraham, A. Oviedo \& E. Willink. 2007. Demographic and quality control parameters of Anastrepha fraterculus (Diptera: Tephritidae) maintained under artificial rearing. Florida Entomologist, 90: 53-57.

Zar, J. H. 1999. Biostatistical Analysis. Prentice-Hall, Inc. Upper Saddle River, NJ. 